\title{
Corrigendum
}

\section{Association between dietary inflammatory index and cardiometabolic risk factors among Brazilian adolescents: results from a national cross-sectional study - CORRIGENDUM}

Pâmela Ferreira Todendi, Rafaela Salla, Nitin Shivappa, James R. Hebert, Julianna Ritter, Felipe Vogt Cureau and Beatriz D. Schaan

(First published online 21 September 2021) DOI: $10.1017 /$ S0007114521003767

The publisher apologises for an error in the final author's name; Beatriz D'Agrord Schaan.

The correct name is Beatriz D. Schaan. 